\title{
Composition and Thrombolytic Susceptibility of Ex Vivo Venous Thromboembolism
}

\section{Samuel A. Hendley}

University of Chicago

Alexey Dimov

Cornell University

Aarushi Bhargava

University of Chicago

\section{Erin Snoddy}

University of Chicago

Daniel Mansour

University of Chicago

Rana O. Afifi

The University of Texas Health Science Center at Houston

Geoffrey D. Wool

University of Chicago

Yuanyuan Zha

University of Chicago

Steffen Sammet

University of Chicago

Zheng Feng Lu

University of Chicago

Osmanuddin Ahmed

University of Chicago

Jonathan D Paul

University of Chicago

Kenneth B Bader ( $\boldsymbol{\sim}$ baderk@uchicago.edu )

University of Chicago

\section{Research Article}

Keywords: Venous thromboembolism, morbidity and mortality worldwide, imaging metrics, treatment outcomes

Posted Date: July 28th, 2021 
DOl: https://doi.org/10.21203/rs.3.rs-739402/v1

License: (c) (1) This work is licensed under a Creative Commons Attribution 4.0 International License. Read Full License 


\title{
Composition and thrombolytic susceptibility of ex vivo venous thromboembolism
}

Samuel A. Hendley ${ }^{1}$, Alexey Dimov ${ }^{2}$, Aarushi Bhargava ${ }^{3}$, Erin Snoddy ${ }^{3}$, Daniel Mansour ${ }^{3}$, Rana O. Afifi ${ }^{4}$, Geoffrey D. Wool ${ }^{5}$, Yuanyuan Zha ${ }^{6}$, Steffen Sammet ${ }^{1,3}$, Zheng Feng Lu ${ }^{1,3}$, Osmanuddin Ahmed $^{3}$, Jonathan D. Paul ${ }^{7}$, Kenneth B. Bader ${ }^{1,3^{*}}$

1University of Chicago, Committee on Medical Physics, Chicago, IL, 60637, USA

2Weill Cornell Medicine, Department of Radiology, New York, NY, 10021, USA

3University of Chicago, Department of Radiology, Chicago, IL, 60637, USA

4University of Texas at Houston, Department of Cardiothoracic and Vascular Surgery, Houston, TX, 77030, USA

5University of Chicago, Department of Pathology, Chicago, IL 60637, USA

6University of Chicago, The Human Immunological Monitoring Facility, Chicago, IL 60637, USA

7University of Chicago, Department of Medicine, Chicago, IL, 60637, USA

*baderk@uchicago.edu

these authors contributed equally to this work

\begin{abstract}
Venous thromboembolism is a significant source of morbidity and mortality worldwide. Catheterdirected thrombolytics is the primary treatment used to relieve critical obstructions, though its efficacy varies based on the thrombus composition. Non-responsive portions of the specimen often remain in situ, which prohibits mechanistic investigation of lytic resistance or the development of diagnostic indicators for treatment outcomes. In this study, thrombus samples extracted from venous thromboembolism patients were analyzed ex vivo to determine their histological structure, lytic susceptibility, and imaging characteristics. A wide range of thrombus morphologies were observed, with a dependence on age and etymology of the specimen. The lytic inhibitor PAI-1 was
\end{abstract}


present in samples, and may contribute to the lytic resistance of venous thrombi. Finally, a correlation was observed between the lytic response of the sample and its magnetic microstructure assessed with a quantitative MRI sequence. These findings highlight the myriad of changes in venous thrombi that may promote lytic resistance, and imaging metrics that correlate with treatment outcomes.

\section{Introduction}

Venous thromboembolism (VTE) is a major health problem that affects 600,000 Americans annually ${ }^{1}$, and up to $10 \mathrm{M}$ worldwide ${ }^{2}$. There is a significant financial burden, with healthcare costs exceeding seven billion dollars each year in the United States associated with VTE ${ }^{3}$. The two primary clinical manifestations of VTE are pulmonary embolism (PE) and deep vein thrombosis (DVT). Pulmonary embolism is the most serious manifestation of VTE, and carries a $25 \%$ mortality rate and a 30-day survival rate of $59 \%{ }^{4}$. It is estimated that $80 \%$ of PE cases originate from deep vein thrombi, primarily in the iliofemoral vasculature ${ }^{5}$. Beside the potential for embolization, there is prevalent morbidity associated with DVT including chronic painful leg swelling and/or venous ulcerations ${ }^{6}$. Phlegmasia cerulea dolens is another disease state secondary to DVT that may result in circulatory compromise ${ }^{7,8}$. Rapid restoration of flow is required for phlegmasia cerulea dolens patients to prevent amputation of the afflicted limb (15\%) or death $(25 \%)^{9,10}$. While not as widely studied as arterial thrombosis, these data illustrate VTE requires effective treatments and screening methods.

Anticoagulation therapy alleviates the symptoms of VTE and prevents thrombus growth ${ }^{11}$. More aggressive approaches are often necessary to prevent the most egregious outcomes of DVT and PE. The standard of care for large vessel recanalization is catheter-directed thrombolytics, which is administered over the course of hours to days ${ }^{12}$. Recombinant tissue plasminogen 
activator (rt-PA) is the principle thrombolytic drug used in the western world ${ }^{13}$. This approach disintegrates fibrin components within the thrombus, and is effective for acute disease ${ }^{14-17}$. Chronic thrombus is a prevalent feature of $\mathrm{VTE}^{18,19}$, and is resistant to rt-PA ${ }^{20}$. Better long-term patient outcomes are observed for when the vessel is fully recanalized ${ }^{21}$, and lytic can be administered up to four days in an attempt to disintegrate residual thrombus ${ }^{12}$. The extended treatment time increases the risk of serious bleeding complications associated with rt-PA ${ }^{22}$, and healthcare costs. Alternative interventions such as mechanical extraction ${ }^{23,24}$ or histotripsy-aided thrombolysis ${ }^{25}$ can be effective for thrombi non-responsive to lytics. The development of methods to identify lytic-resistant, chronic thrombi a priori will expedite the use of these alternative treatment schemes or new approaches for chronic VTE strategies.

The precise mechanism by which chronic VTE develop resistance to lytic therapy remains elusive. Animal models do not fully replicate the clotting cascade of humans ${ }^{26-29}$, and traditional treatment methods leave the sample in situ ${ }^{30}$. The advent of venous-specific thrombectomy devices enables access to VTE samples for characterization ${ }^{31-33}$. In this study, qualitative and quantitative histological analysis was conducted to assess formed elements of VTE specimen ex vivo. Whole sample imaging markers of iron (marker of erythrocytes) via quantitative MRI susceptibility mapping and stiffness (marker of extracellular thrombus structure) via ultrasound shear-wave elastography were collected. Finally, an in vitro assay was conducted to determine the lytic susceptibility of the specimen. Primary findings indicate that collagen increased and erythrocytes decreased with thrombus age, and plasminogen activator inhibitor 1 is a prevalent feature of VTE. Further, the lytic susceptibility of a thrombus correlated with its magnetic susceptibility assessed via MRI. The observed changes in clot structure reflect the complex mechanisms by which VTE 
microstructure may develop lytic resistance, and these mechanisms can be gauged using imaging metrics.

\section{Results}

Approach

Analysis was performed on 26 venous thrombi aspirated acutely (12 deep vein thrombi and 14 pulmonary emboli). Details for the patient demographics are presented in Supplemental Table 1. Samples were sectioned to conduct analysis via of the formed elements (histology, immunohistochemistry, and immunofluorescence), imaging features (MRI and ultrasound), or lytic susceptibility. For histological studies, three sections from each specimen were stained to highlight erythrocytes, fibrin, collagen, and platelets for a total of 234 total images analyzed. A subset of the collected samples four were subjected to immunofluorescence to quantify the colocation and presence of formed elements. Separate sections were embedded in agarose gel for imaging analysis. Ultrasound shear wave elastography was used to assess the elastic modulus of the sample, and the MRI sequence quantitative susceptibility mapping to gauge the fraction of erythrocytes in the sample. Finally, an in vitro assay was conducted to determine lytic susceptibility by exposing the specimen to plasma and rt-PA. Mass loss was used as a metric to quantify the success of catheter-directed thrombolysis.

\section{Qualitative assessment of thrombus composition}

The fraction of fibrin, collagen, erythrocytes, and platelets observed in samples is shown in Fig. 1. A wide range in composition was noted, with fibrin and erythrocytes as the primary thrombus components ( $52 \pm 26 \%$ and $35 \pm 23 \%$ of thrombus area, respectively). Collagen constituted less 
than $2 \%$ of the thrombus area on average, though a maximum of $\sim 60 \%$ was observed in one sample (Fig. 2). When present, collagen was observed in discrete clusters those prevalence increased towards the edge of the thrombus (i.e. towards the thrombus/vessel wall interface). Platelets were observed as a prevalent feature as well in samples, and their density also increased towards the edges of the sample. Erythrocytes tended to be located near the center of the thrombus and were less likely to be observed along the edges. Fibrin was uniform across the samples.

Analysis was also conducted to categorize the samples based on anatomic location of extraction (Fig. 1). Mean values for the area of collagen, fibrin, erythrocytes, and platelets were not significantly different between PE and DVT samples. However, the sample-to-sample variability in fibrin and erythrocytes was reduced in DVT samples in comparison to PE.
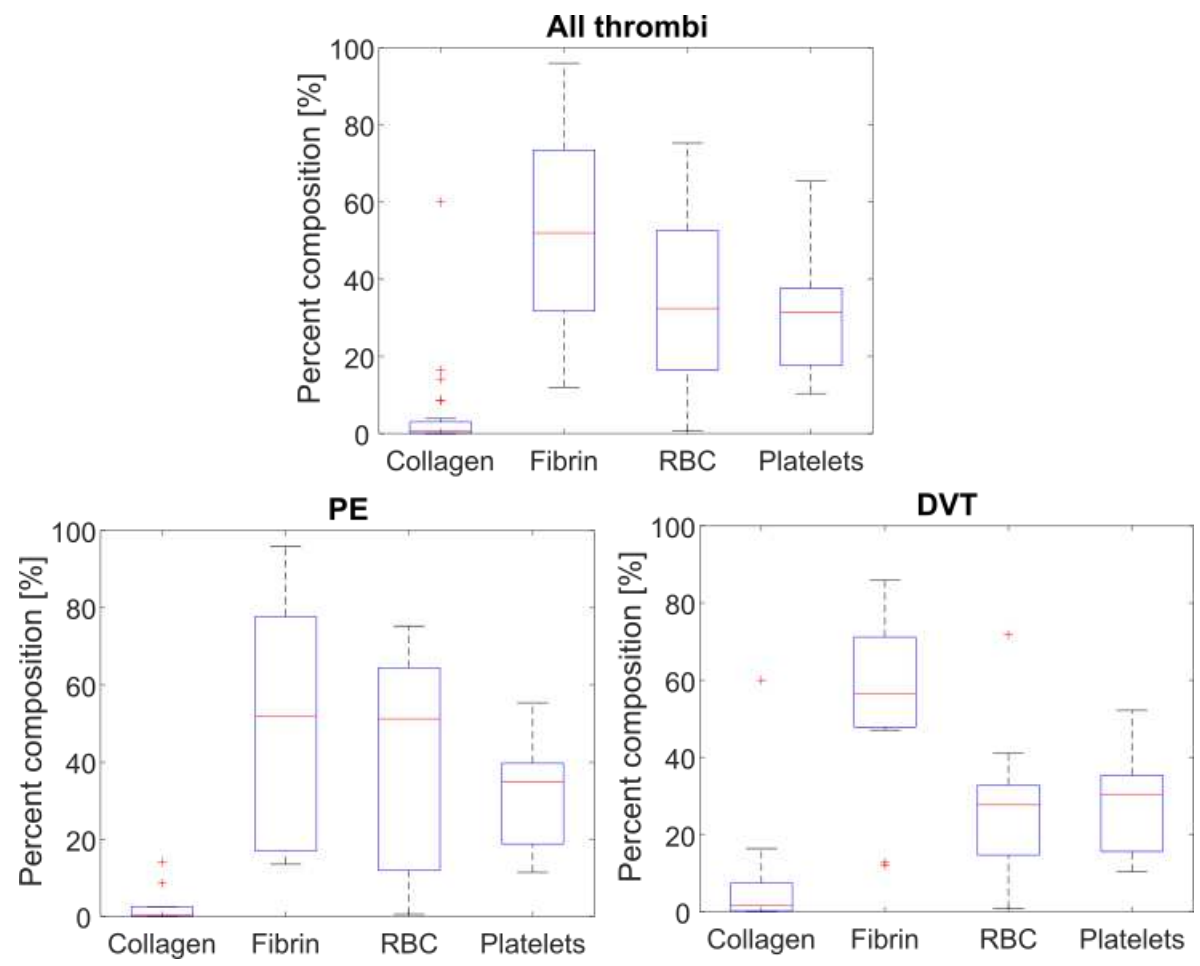

Figure 1: The fractional area of collagen, fibrin, platelets, and erythrocytes (RBC) present in (top) all analyzed VTE specimen, (bottom left) pulmonary emboli, and (bottom right) deep vein thrombi. Red crosses indicate outliers and horizontal red lines indicate median values. The top and bottom portions of the blue box represent the $25^{\text {th }}$ and $75^{\text {th }}$ percentiles, respectively, and whiskers extent to the data points not considered outliers. 


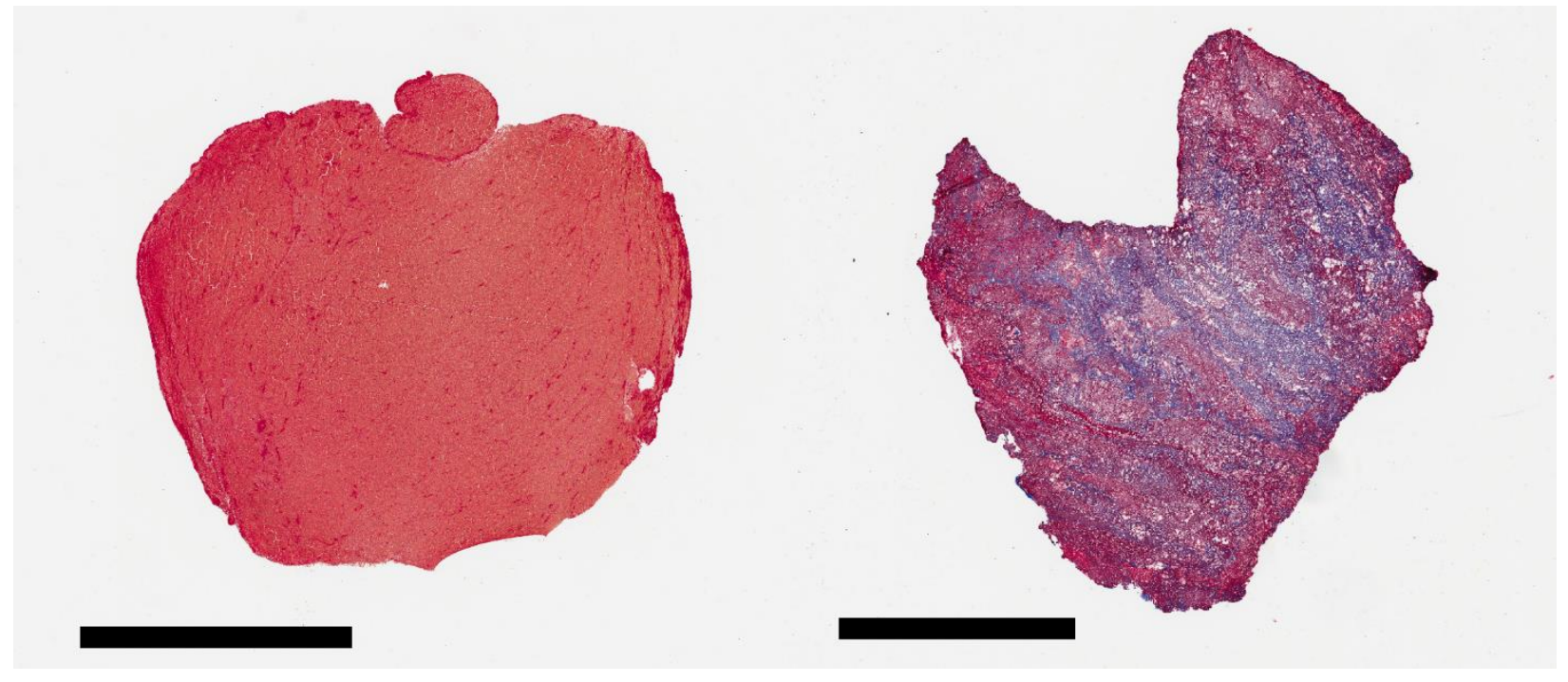

Figure 2: Observed patient-to-patient variability in thrombus collagen composition $(0.06 \%$ by area for left vs. $60 \%$ for right), which appears blue in the Masson's trichrome stain. The scale bars indicate $2 \mathrm{~mm}$.

Trends between collagen, fibrin, erythrocytes, and platelets are shown in Fig. 3 to gauge the interdependence of formed elements. The strongest correlation was a negative relationship between fibrin and erythrocytes $\left(\mathrm{R}^{2}=0.83 ; \mathrm{p}<0.01\right)$. A medium-strength positive correlation was observed between fibrin and platelets $\left(\mathrm{R}^{2}=0.43 ; \mathrm{p}<0.01\right)$. Significant correlations were also noted between erythrocytes and platelets (negative) $\left(\mathrm{R}^{2}=0.26 ; \mathrm{p}=0.01\right)$, erythrocytes and collagen (positive) $\left(\mathrm{R}^{2}=0.22 ; \mathrm{p}=0.02\right)$, and fibrin and collagen (positive) $\left(\mathrm{R}^{2}=0.20 ; \mathrm{p}=0.02\right)$. There was no correlation observed between collagen and platelets $\left(R^{2}=0.04 ; p=0.3\right)$. 

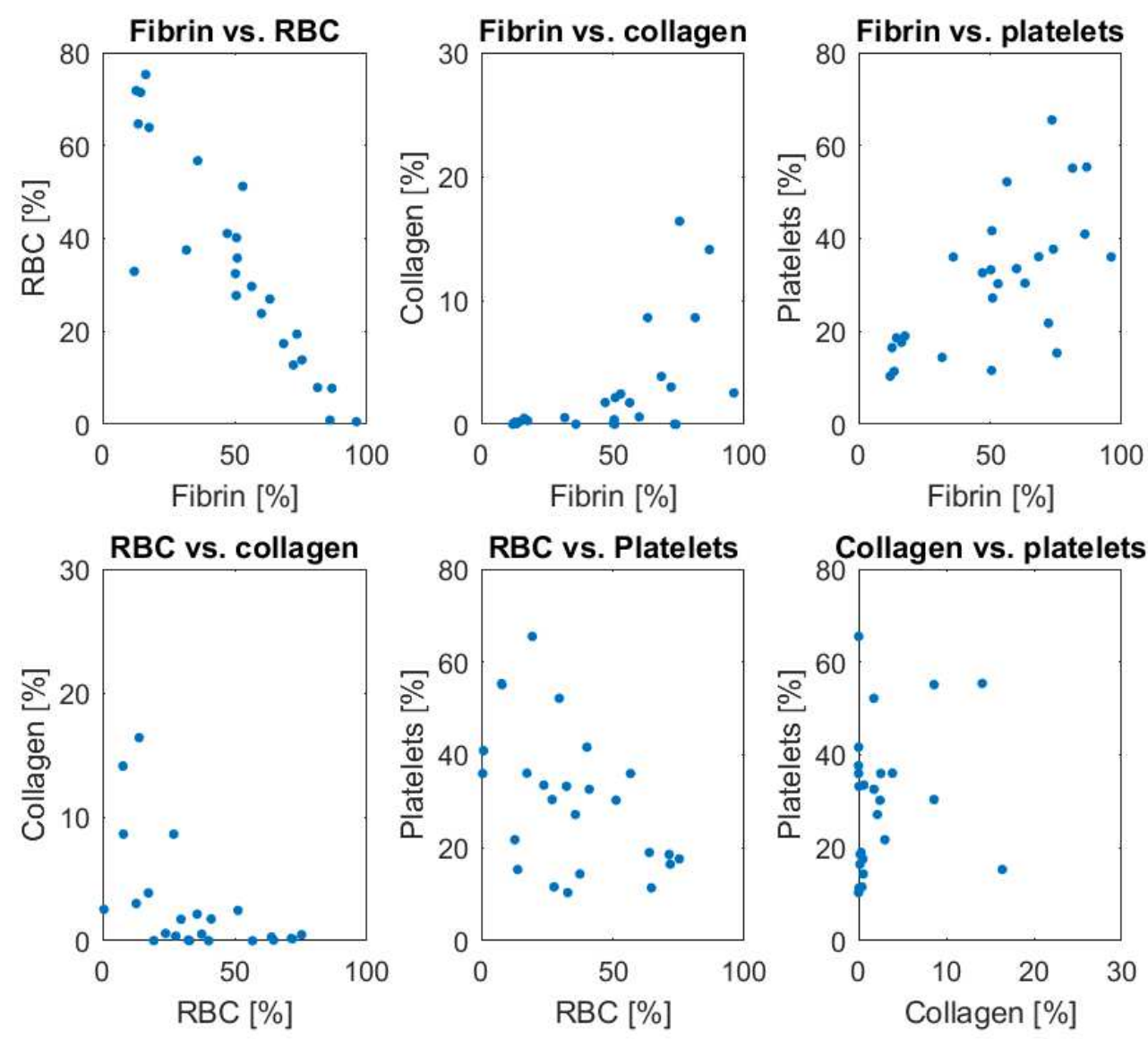

Fibrin [\%]

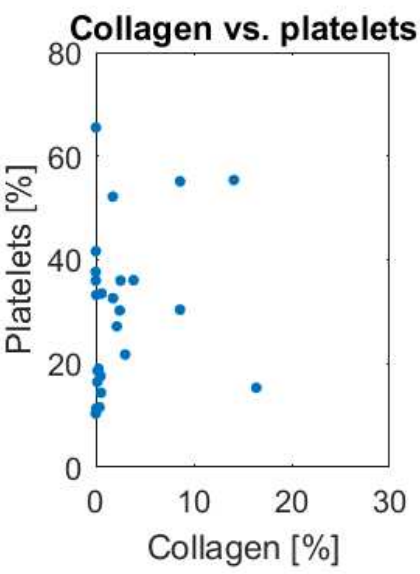

Figure 3: Trends in thrombus composition for fibrin, collagen, red blood cells (RBCs), and platelets.

\section{Change in thrombus structure with age}

Prior studies indicated significant structural remodeling of VTE after seven days ${ }^{34,35}$. Figure 4 depicts the histological composition of VTE less than and greater than seven days old. The thrombus age was designated based on the time between the onset of patient symptoms and the mechanical thrombectomy procedure. Wilcoxon ranked sum tests indicated that thrombi one or more weeks old were composed of more collagen $(p=0.03)$ and fewer erythrocytes $(p=0.02)$ compared to acute specimen. No changes were observed in the fraction of fibrin $(\mathrm{p}=0.19)$ or platelets $(\mathrm{p}=1.00)$ with thrombus age. 

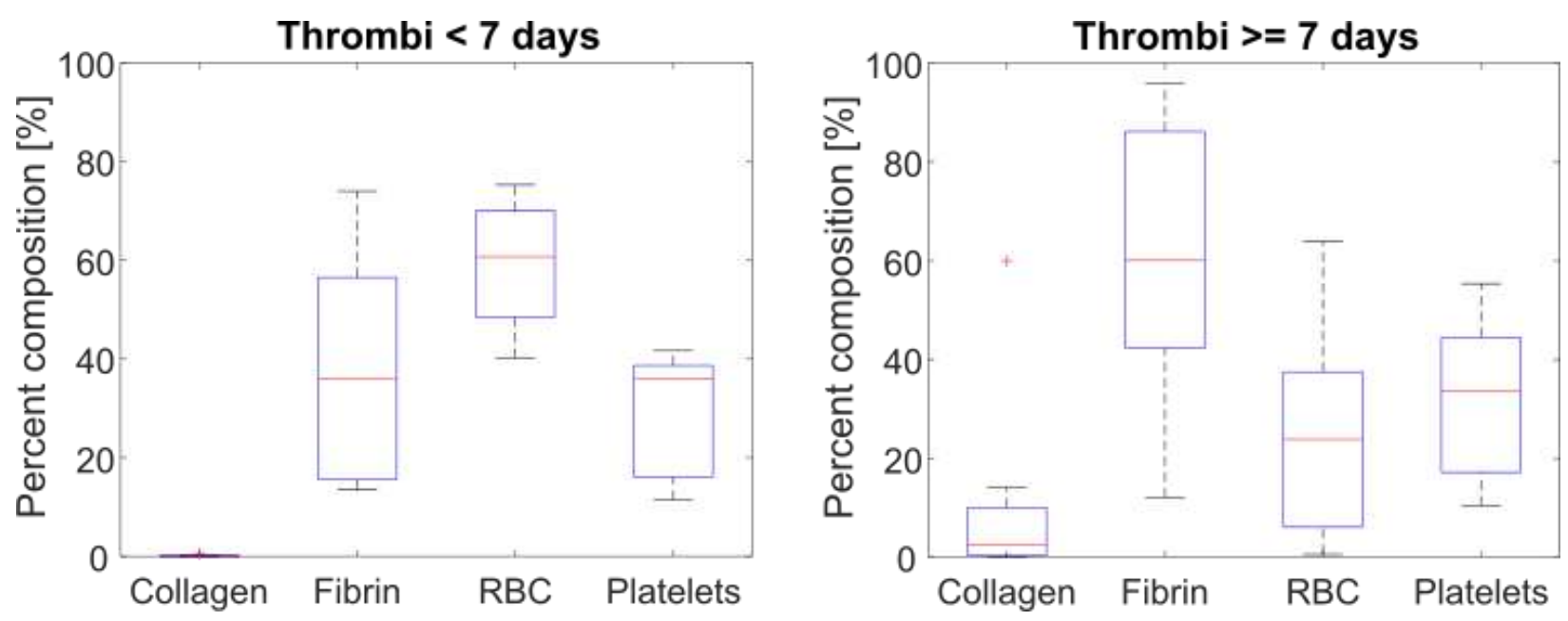

Figure 4: Thrombus composition for acute ( $<7$ days old) and chronic ( $\geq 7$ days old) thrombi.

\section{Quantification of thrombus composition}

Figure 11 depicts representative immunofluorescence staining and imaging to quantify the thrombus composition. Collagen, fibrin, and platelets were observed in close proximity (i.e. within $50 \mu \mathrm{m})$. Erythrocytes were observed in areas of low fibrin concentration. Plasminogen activator inhibitor-1 (PAI-1) was co-located with a subpopulation of the nucleated cells present in the sample (Fig. 6) and erythrocytes (Fig. 5). Multiple markers of neovascularization were tested to indicate vessel wall remodeling (vascular endothelial growth factor receptor 1,VEGFR-1, and cluster of differentiation 31, CD31), but were not observed. 


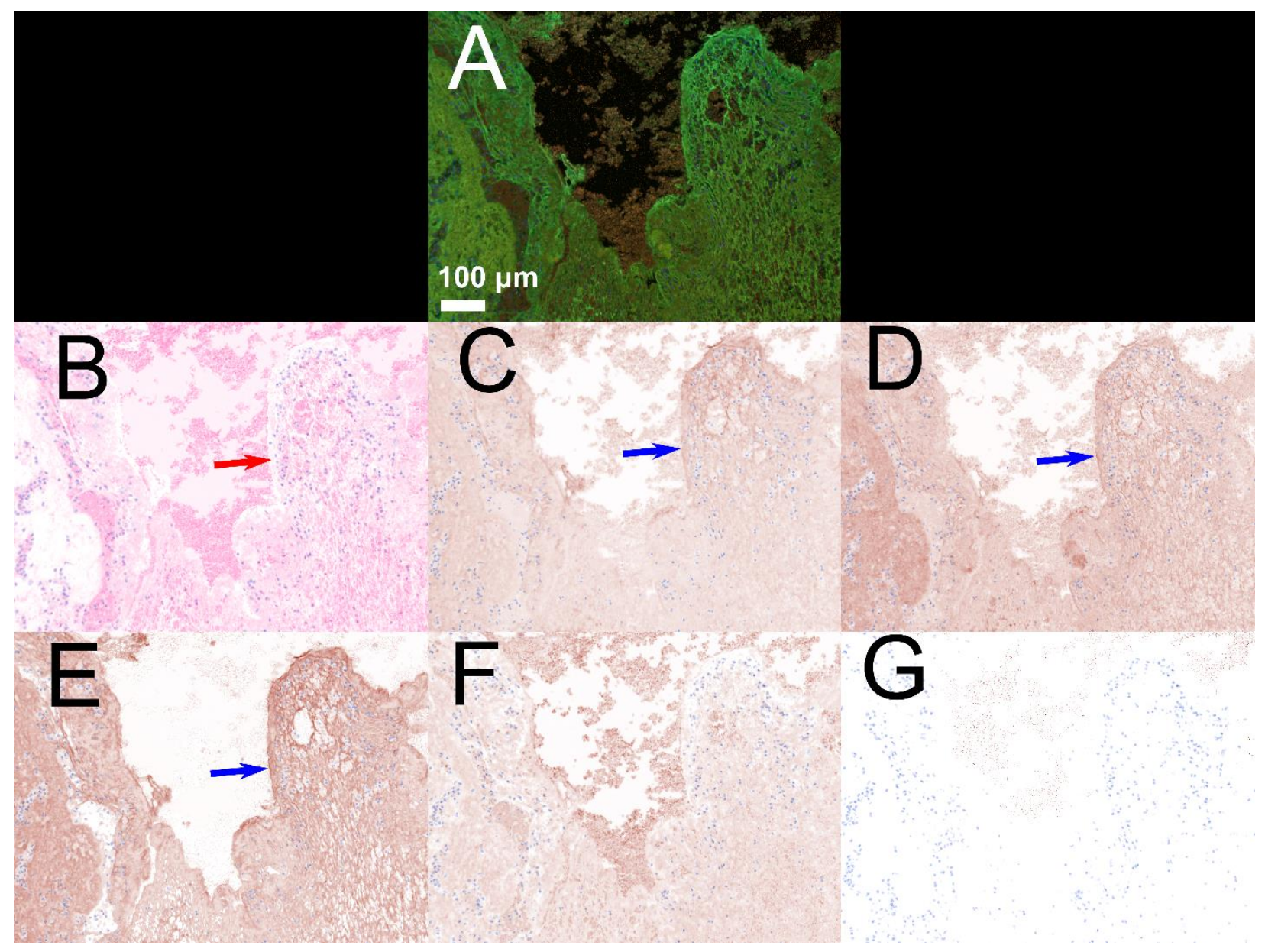

Figure 5: Representative immunofluorescent data collected from VTE. (A) Combined overlay and thrombus components analyzed using immunofluorescence, (B) erythrocytes, (C) platelets, (D) collagen, (E) fibrin, (F) PAI-1 and (G) VEGR-1. Images B-G are spectral deconvolutions of the single image A. The scale bar in image A applies to all images. Blue arrows indicate the colocation of fibrin, collagen, and platelets, respectively. The red arrow indicates a fibrin-rich border that contains almost no red blood cells. Antibody information can be found in Supplemental Table 1 . 


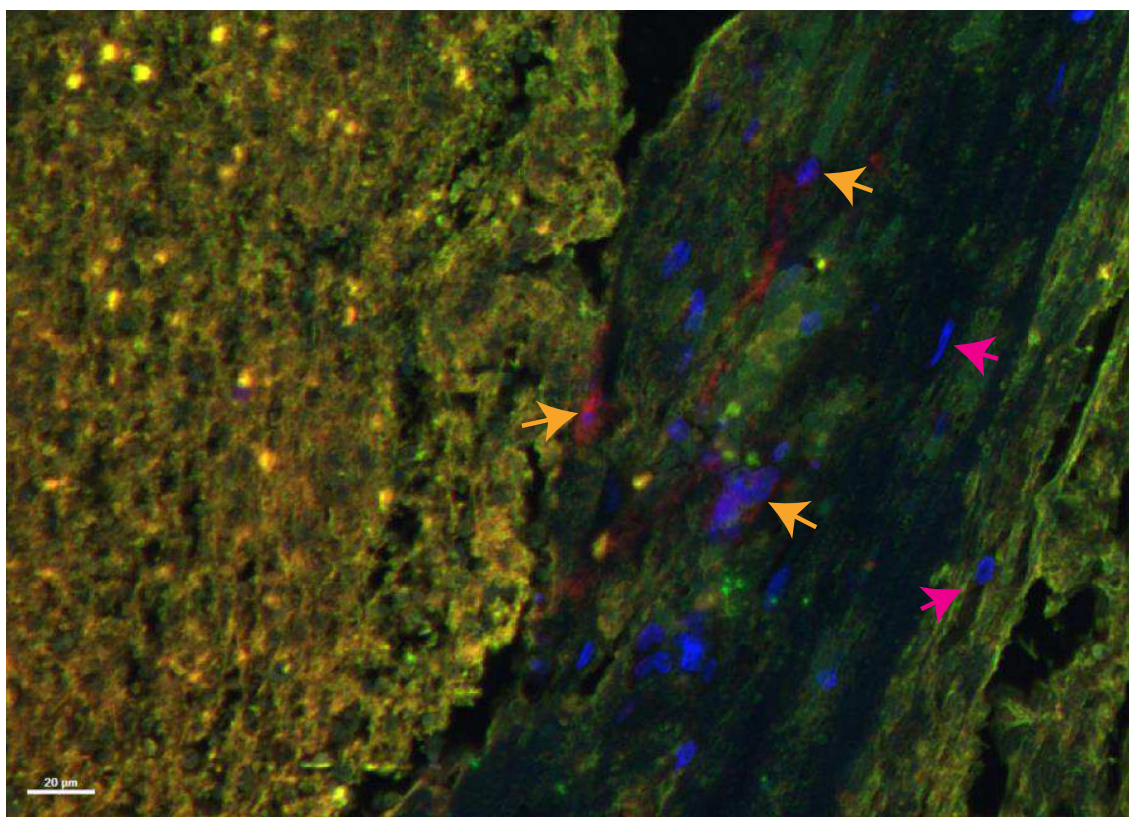

Figure 6: Example of nucleated cells present in the sample (blue) along with PAI-1 (red). Orange arrows indicate nucleated cells that are co-located with PAI-1, whereas magenta arrows indicate nucleated cells within the clot not associated with PAI-1.

\section{Thrombus stiffness}

Significant variation was observed in the stiffness of samples, with elastic moduli varing from $53 \mathrm{kPa}$ to $122 \mathrm{kPa}$. There was also significant intrathrombus stiffness variation (Supplemental Fig. 1), indicating the heterogeneity of the analyzed VTE. No trends were indicated between the stiffness and thrombus composition assessed via histological stains, or between sample stiffness and its susceptibility to rt-PA.

\section{Assessment of hemoglobin content}

The findings in this study and others indicate higher erythrocyte content for acute thrombi relative

to chronic disease. Hemoglobin within erythrocytes leads to changes in the local magnetic susceptibility, which can be tracked with a quantitative susceptibility-weighted MRI sequence. 
The specimen collected in this study had heterogenous magnetic susceptibility (Supplemental Fig. 2) due to concentrated zones of high and low iron content. Positive-pixel QSM values indicative of erythrocytic content were tabulated for each sample and correlated with its response to lytic ${ }^{36}$. A significant slope was observed between the magnetic susceptibility of the sample and its disintegration under the action of rt-PA (Fig. 7, $\rho=0.90, \mathrm{p}<0.01$ ).

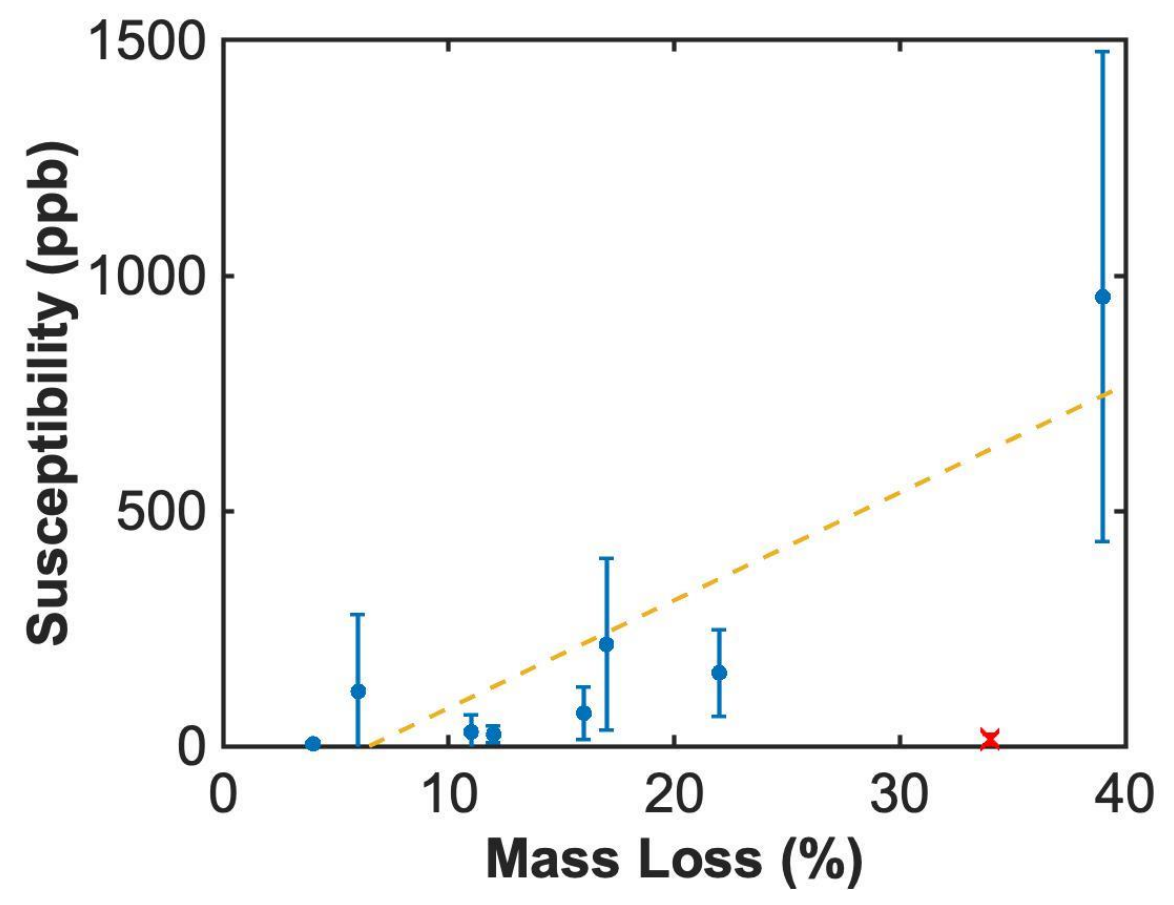

Figure 7: Comparison of clot mass loss vs. quantitative susceptibility map data ( $\boldsymbol{\rho}=0.90, \mathrm{p}<0.01, \mathrm{~N}=9$ samples). Error bars represent the range of positive QSM values observed within the sample. One sample was highly heterogenous (red cross), and gross observation indicated strong variation between the portion of that specimen that was subjected to rt-PA and the portion subjected to QSM analysis. The dashed yellow line is a least-squares best fit to the data $\left(R^{2}=0.77\right)$.

\section{Discussion}

A primary goal for this study was to assess the composition of VTE. To date, the composition of venous thrombi have been gauged based on animal models or postmortem samples ${ }^{37,38}$. Analysis 
of ex vivo VTE specimen is limited as current approaches treat the thrombus in situ or destroy its structure during removal. The recent advent of venous-specific mechanical thrombectomy devices allowed for the removal of thrombi while still preserving the overall structure ${ }^{31-33}$.

Fibrin and erythrocytes were the primary thrombus components observed in these specimen, consistent with the observations of other studies ${ }^{33}$. Compared to a recent investigation of venous thrombus composition ${ }^{39}$, the prevalence of fibrin $(\sim 53 \%$ vs. $35 \%)$ and platelets $(\sim 30 \%$ compared to $4 \%$ ) were increased and erythrocytes decreased ( $\sim 35 \%$ vs. $63 \%)$. There may be multiple reasons for the discrepancy between these two studies. Here, formed elements were quantified across the entire area of the thrombus with immunohistochemistry (which is semiquantitative), whereas only subsections of the thrombus were analyzed using scanning electron microscopy in the prior study. Samples here were obtained via venous-specific, minimally-invasive mechanical thrombectomy devices, which may not extract structures adherent to the vessel wall. Open thrombectomy was performed for retrieval of other samples. Regardless, the differences observed indicate a diverse range of VTE composition.

Interestingly, platelets were a significant component of these VTE samples. The standard convention is that venous thrombi are platelet poor, and primarily comprised of erythrocytes entangled in fibrin (i.e. a "red" thrombus) ${ }^{40}$. In contrast, fibrin is through to form as a result of platelet aggregation in arterial thrombi, resulting in minimal erythrocyte content (i.e. "white" thrombus). Platelets contribute to retraction of the thrombus ${ }^{41}$, which is associated with reduced rt-PA susceptibility ${ }^{42}$. Even treatments that do not require intervention may be altered by the prevalence of platelets, indicating that antiplatelet medications typically reserved for arterial thrombi may be appropriate for a subset of $\mathrm{VTE}^{43}$. Regardless, these findings indicate there may be less of a distinction between arterial and venous thrombi. 
Thrombus age was an important factor in the thrombus composition, including a reduction in erythrocytes for chronic disease. Prior findings indicate erythrocytes are damaged and removed as the thrombus ages ${ }^{44}$, consistent with the findings here. There are multiple types of erythrocytes ${ }^{45}$, with polyhedrocytes being the most prevalent form found in venous thrombi. The polyhedrocyte form is promoted through platelet-driven retraction of the thrombus, and is associated with a reduced propensity for embolization. The prevalence of platelets observed in these studies suggest polyhedrocytic erythrocytes for these samples, though further studies are needed to assess the precise form.

The extracellular structure of chronic samples was also changed via increases in collagen deposition. The infiltration of fibroblasts into the thrombus is the primary cause of collagen deposition. The process occurs over the course of several days ${ }^{46,47}$, consistent with the lack of observed collagen for acute samples. A hypothesized mechanism of lytic resistance for chronic thrombi is a change in extracellular structure ${ }^{48,49}$. Because of its fibrin-specific protolysis by plasmin $^{50}$, rt-PA is not effective for collagenous structures. Fibrin was more prevalent than collagen even in chronic samples (on average $\sim 60 \%$ vs. $5 \%$ of thrombus area, Fig. 4 ), in agreement with another recent analysis of $\mathrm{VTE}^{33}$. The method of specimen extraction may contribute to the degree of collagen observed in samples. Fibroblasts are recruited to the thrombus through vesselwall derived factors, and collagen elements adherent to the vascular wall may not have been retrieved. Nevertheless, these findings suggest fibrin is a primary component of chronic thrombus, and collagen deposition constitutes a secondary effect on the resistance of chronic thrombi. Lytic may therefore still be an effective method for recanalization provided it can be distributed throughout the chronic thrombus extent. 
Though multiple markers were tested, there was no indication of neovascularization within the thrombus. The presence of new vasculature is attributed to remodeling of the venous wall ${ }^{34}$, and is located near the thrombus/vessel interface. Plasminogen activator inhibitor 1 (PAI-1) was observed in samples, a protease inhibitor that acts to quench rt-PA activity and halt fibrinolysis, and may be a key factor in VTE lytic resistance. A subset of nucleated cells within the samples were co-located with PAI-1, though the precise cell type was undetermined. Prior studies indicate that PAI-1 is co-located with neovascularization ${ }^{51-53}$, though there was no indication of either VEGFR-1 or endothelial cells in theses samples. Leukocytes in the form of macrophages also produce PAI- ${ }^{54}$, though further work is needed to quantify the precise progenitor for these samples. After generation, PAI-1 is retained within platelets prior to activation. The VTE samples examined here were platelet rich, and may contribute to prevalence of PAI-1 and lytic resistance of samples. Inhibitors for PAI-1 are readily available to reestablish fibrinolysis ${ }^{55}$. When coadministered with lytic therapy, PAI-1 inhibitors may provide a means to ensure disintegration for more chronic VTE.

Etiology was also noted to affect the thrombus structure. Less variability in fibrin and erythrocytes was noted in DVT compared to PE (Fig. 1). The concentration of erythrocytes was noted as a marker of thrombus age (Fig. 4). Over $80 \%$ of pulmonary emboli originate as deep vein thrombi ${ }^{5}$. Embolized chronic DVT may serve as a nidus for acute clots, thereby creating a more heterogeneous specimen for $\mathrm{PE}$ and therefore the observed increased range of erythrocytes compared to DVT samples.

A secondary goal of this study was to determine the relationship between quantitative imaging metrics (i.e. QSM or ultrasound shear wave elastography) and thrombolytic efficacy. Current imaging metrics for VTE rely on characteristics related to perfusion or stiffness, but lack 
information on the thrombus microenvironment responsible for its response to lytic therapy. Magnetic resonance imaging is well-suited to measuring the microstructure of thrombi ${ }^{56}$, including erythrocyte content. A significant trend was noted between magnetic susceptibility and thrombolysis (Fig. 7). Deoxygenated hemoglobin within erythrocytes has a high magnetic susceptibility $^{57}$, and is the primary source of contrast for QSM in these samples ${ }^{58}$. The correlation between QSM and thrombolysis indicates lytic susceptibility increases with the concentration of erythrocytes within the thrombus. This agrees with the known relationship between thrombus age and lytic susceptibility and with our observation that older thrombi contain fewer erythrocytes compared to acute thrombi.

Prior studies have indicated that increased stiffness is a hallmark of chronic thrombi ${ }^{59,60}$, and that stiffness is inversely correlated with lytic susceptibility ${ }^{42}$. No trends were observed here between the elastic modulus of the samples and either thrombus composition or thrombolysis metrics. A large variations in thrombus stiffness was observed, with elastic moduli varying by more than $80 \mathrm{kPa}$ within a single sample. The average stiffness may not be a good metric because of the inhomogeneities seen in these samples. The ultrasound images are inherently twodimensional, and volumetric assessment of the sample stiffness may provide a more applicable comparison to bulk measurements such as mass loss. Because of its inability to penetrate the lung, ultrasound may not be a viable modality to gauge PE in vivo.

There are a number of limitations to this study that limit generalizability of the finding. Samples may be altered by the mechanical extraction procedure. Thrombolytic susceptibility of samples was tested using a systemic lytic administration scheme, whereas catheter-infusion of the lytic within the thrombus is a primary treatment scheme for VTE. Biological factors beyond composition such as blood flow may affect the lytic profile in situ compared to ex vivo ${ }^{61}$. Due to 
the retrospective nature of this study, sample ages were estimated based on the onset of symptoms and may not reflect the true thrombus age given dependence on reliability patient reporting. To limit the time required for data collection and mitigate sample degradation, separate sections of the thrombus were used for histology, imaging, and assessment of lytic susceptibility. Gross inspections were conducted for samples subjected to each type of analysis, though specimen were heterogenous. Due to the limited size of some of the thrombus samples, not all thrombi could be analyzed with our full testing panel (i.e. histological analysis, thrombolysis, QSM, and elastography). Histological analysis was conducted to determine the presence of fibrin and erythrocytes. The precise phenotype of these elements was not assessed, which can affect relevant properties of the thrombus (e.g lytic susceptibility ${ }^{39,62}$ ). Nevertheless, the observed trends between QSM pixel values and lytic response indicates a potential means to prescreen patients for thrombolytic therapy and thus decrease treatment risk.

\section{Methods}

\section{Thrombus collection}

As part of the standard of care, thrombi were collected from patients undergoing mechanical thrombectomy procedures at the University of Chicago Medical Center and the University of Texas Health Science Center. Demographics for patient data are shown in Supplemental Table 1. Thrombi were subjected to analysis following local internal review board approval and informed consent (University of Chicago IRB \#18-0179). Extracted samples were stored in saline solution and processed for analysis within 24 hours of collection. A total of 26 thrombi were examined, of which 12 were deep vein thrombi from iliocaval veins, and 14 were pulmonary emboli. Samples 
were sectioned, with representative subsections being subjected to histology, imaging, or lytic response. Not all samples were large enough to conduct each set of analyses (26 histology samples, 9 thrombolysis samples, 9 QSM samples, 12 elastography samples).

\section{Assessment of clot structure via histology}

Histology was used for qualitative analysis of the sample structure. Immediately after collection, thrombi were sectioned to $\sim 3 \mathrm{~mm}$ thickness and submerged in $10 \%$ formalin for 24 hours. After fixation, thrombus sections were submitted to the University of Chicago Human Tissue Resource Center for paraffin-embedding, sectioning ( $5 \mu \mathrm{m}$ thickness), and histochemical staining. Three stains were used: Hematoxylin \& Eosin (H\&E, Tissue-Tek Prisma H\&E Stain Kit \#1, Sakura Finetek, USA), Masson's Trichrome (Trichrome Staining Kit, Hoffmann-La Roche, Switzerland), and anti-CD-61 immunohistochemistry (CD61, Platelet Glycoprotein IIIa, Agilent, USA). Three sections were analyzed for each sample to gauge heterogeneity.

Stained specimen were scanned at 20x magnification using a ScanScope XT scanner (Leica Biosystems, Germany). Digitized slides were viewed using ImageScope software (Leica Biosystems, Germany), and were analyzed using the Positive Pixel Count algorithm (Leica Biosystems, Germany) to identify red blood cells and fibrin in H\&E stains, collagen in Masson's Trichrome stains, and platelets in CD61 stains ${ }^{63}$. The number of pixels that fell within the designated color parameters (i.e. thrombus component) were quantified and binned into intensity ranges: negative, weak positive, positive, or strong positive. Color thresholds were evaluated and accepted by a board-approved pathologist, as depicted in Supplemental Fig. 3. 
Four samples were also subjected to immunofluorescent analysis to quantitatively assess formed elements. Fluorescent antibodies were used to examine the co-location of fibrin (MABS2155, Millipore Sigma, USA), collagen (Maine medical center research institute, USA), platelets (CD-61, M075301-2, Agilent, USA), vascular endothelial growth factor (VEGF, ab32152, abcam, USA), and plasminogen-activator inhibitor-1 (PAI-1, ab125687, abcam, USA). Erythrocytes were examined utilizing their innate autofluorescence and did not require staining. Two samples were stained to gauge the co-expression of PAI-1 and the endothelial cell marker CD31 (ab28364, abcam, USA). Samples were stained using an Opal 7-color manual IHC kit (Akoya Biosciences, USA) and were scanned using a Vectra Polaris whole slides scanner (Akoya Biosciences, USA). Additional antibody information can be found in Supplemental Table 2.

\section{Assessment of thrombolytic susceptibility}

Human fresh-frozen type O plasma was obtained from a blood bank (Vitalant, Chicago, IL), thawed and aliquoted in $30 \mathrm{~mL}$ increments, and stored at $-80^{\circ} \mathrm{C}$ before use ${ }^{64}$. Alteplase was obtained as a lyophilized powder (Activase, Genetech, San Francisco, CA, USA), mixed with sterile water to a concentration of $1 \mathrm{mg} / \mathrm{mL}$, and stored at $-80^{\circ} \mathrm{C}$ before use. Ex vivo thrombi were sectioned into $\sim 1 \mathrm{~cm}$ segments and were exposed to either plasma and rt-PA $(2.68 \mu \mathrm{g} / \mathrm{mL}$, consistent with pharmacomechanical VTE treatment ${ }^{65}$ ) or plasma alone (control). To account for the heat-dependent enzymatic activity of rt-PA, thrombus sections were placed in individual latex containers filled with $15 \mathrm{~mL}$ of human plasma with or without rt-PA and were submerged in a water tank heated to physiologic temperature $\left(37^{\circ} \mathrm{C}\right)$. The percent change in mass of the sample was tabulated for each sample. Physical manipulation of the thrombi may affect the mass of the clot. To account for these factors, thrombus lytic susceptibility was reported as:

$$
M_{\text {residual }}=M_{\text {lytic }}-M_{\text {control }}
$$


where $M_{\text {lytic }}$ is the percent mass loss for clots exposed to lytic and plasma, and $M_{\text {control }}$ is the percent mass loss for clots exposed to plasma alone.

\section{Preparation of samples for imaging}

Samples were embedded in $1.5 \% \mathrm{w} / \mathrm{v}$ low gelling temperature agarose before imaging as follows. Agarose was heated until clear, transferred to an ultrasonic water bath set to $40{ }^{\circ} \mathrm{C}$, and was degassed for 30 minutes. After degassing, the agarose was cooled to $37^{\circ} \mathrm{C}$ and then was poured over the thrombus sample. Any bubbles in the agarose were moved outside of the field of view of the thrombus.

\section{Quantitative susceptibility mapping}

Erythrocytes, a primary component of thrombi, can cause significant alterations of the magnetic susceptibility of a sample. Quantitative susceptibility mapping (QSM) is an MRI sequence that maps the sample magnetization. Paramagnetic tissues appear hyperintense in QSM images, and diamagnetic tissues appear hypointense ${ }^{58}$. A clinical 3 Tesla (3T) MR system (Ingenia dStream, Philips Healthcare, Best, The Netherlands) with a body transmit coil and a 16-channel head-andneck receive coil was used to collect QSM images of the agarose embedded samples. A threedimensional gradient echo recall sequence was employed to acquire multiple echoes. Utilizing the real and imaginary portions of the received signals, magnetic susceptibility was computed via the

morphology enabled dipole inversion (MEDI) pipeline ${ }^{66,67}$. Thrombi were contoured by hand to exclude background QSM values. Positive QSM values (i.e. ppb > 0) were tabulated to gauge the concentration of erythrocytes in VTE samples ${ }^{36}$. This technique exploits the paramagnetic nature of hemoglobin while excluding highly diamagnetic tissues. 


\section{Assessment of clot stiffness via ultrasound elastography}

Ultrasound elastography was used to assess the stiffness of the agarose-embedded ex vivo samples ${ }^{68}$. Ultrasound images were acquired with a linear array transducer (GE Ultrasound Transducer 9L, $9 \mathrm{MHz}$ nominal frequency) and Logiq E9 scanner (GE Healthcare, Chicago, IL, USA). The scanner exam protocol "Small Parts" was utilized. Based on the approximate depth of the sample, a scan depth of 2 to $4 \mathrm{~cm}$, and $9 \mathrm{MHz}$ frequency setting provided good resolution of the sample. Standard B-mode images were used to visualize the cross section of the thrombus (e.g. orthogonal to its in situ vascular orientation), and a shear wave elastography images were used to map the elastic modulus. Three images (e.g. three measurements of elastic modulus) were recorded, and the average elastic modulus tabulated for each sample. For each section, regions-ofinterest $\sim 4 \mathrm{~mm}$ in diameter were selected to quantify the elastic modulus depending on the diameter of the thrombus (mean diameter $0.7 \mathrm{~cm}$, range $0.3-1.4 \mathrm{~cm}$ ). To avoid surface waves along the thrombus/agarose interface, regions-of-interest were not acquired near the edge of the sample.

\section{Statistical analysis}

Statistical analysis was performed using the MATLAB Statistical Toolbox (The Mathworks, Natick, MA, USA). Correlations were quantified using the Pearson's correlation coefficient. Wilcoxon ranked-sum tests were used to compare the composition of thrombi across two age groups and to compare the composition of PE and DVT. 


\section{Data Availability}

The data generated from this study are available from the authors upon reasonable request.

\section{Ethics Declaration}

All experimental protocols were approved by the local internal review board and informed consent (University of Chicago IRB \#18-0179). All methods were carried out in accordance with the relevant guidelines and regulations. Prior to collecting thrombus samples for analysis, informed consent was obtained. These thrombi were extracted from patients mechanically as part of the standard of care and independent from this study.

\section{References}

1. Aparicio, H. J. et al. Heart Disease and Stroke Statistics-2021 Update A Report from the American Heart Association. Circulation (2021). doi:10.1161/CIR.0000000000000950.

2. Raskob, G. E. et al. Thrombosis: A major contributor to global disease burden. Arterioscler. Thromb. Vasc. Biol. 34, 2363-2371 (2014).

3. Grosse, S. D. Incidence-based cost estimates require population-based incidence data. A critique of Mahan et al. Thromb. Haemost. 107, 192-193 (2012).

4. Go, A. S. et al. Heart Disease and Stroke Statistics--2013 Update: A Report From the American Heart Association. Circulation 127, e6--e245 (2013).

5. Fleck, D. et al. Catheter-directed thrombolysis of deep vein thrombosis: Literature review and practice considerations. Cardiovasc. Diagn. Ther. 7, S228-S237 (2017). 
6. Kahn, S. R. The post-thrombotic syndrome: progress and pitfalls. Br. J. Haematol. 134, 357-365 (2006).

7. Lin, S. C., Mousa, A., Bernheim, J. \& Dayal, R. Endoluminal Recanalization in a Patient with Phlegmasia Cerulea Dolens Using a Multimodality Approach. Vasc. Endovascular Surg. (2005).

8. Oguzkurt, L., Ozkan, U., Gulcan, O., Koca, N. \& Gur, S. Endovascular treatment of acute and subacute iliofemoral deep venous thrombosis using manual aspiration thrombectomy: long-term result of 139 patients from a single center. Diagnostic Interv. Radiol. (2011).

9. Patel, N. H., Plorde, J. J. \& Meissner, M. Catheter-directed thrombolysis in the treatment of phlegmasia cerulea dolens. Ann. Vasc. Surg. 12, 471-475 (1998).

10. Hood, D. B., Weaver, F. A., Modrall, J. G. \& Yellin, A. E. Advances in the treatment of phlegmasia cerulea dolens. Am. J. Surg. (1993).

11. Wells, P. S., Forgie, M. A. \& Rodger, M. A. Treatment of Venous Thromboembolism. JAMA 311, 717 (2014).

12. Chen, J. X., Sudheendra, D., Stavropoulos, S. W. \& Nadolski, G. J. Role of Catheterdirected Thrombolysis in Management of Iliofemoral Deep Venous Thrombosis. RadioGraphics 36, 1565-1575 (2016).

13. Vedantham, S., Piazza, G., Sista, A. K. \& Goldenberg, N. A. Guidance for the use of thrombolytic therapy for the treatment of venous thromboembolism. J. Thromb. Thrombolysis 41, 68-80 (2016). 
14. Horne III, M. K. \& Chang, R. Thrombolytic Therapy for Deep Venous Thrombosis? JAMA 282, 2164-2166 (1999).

15. Enden, T. et al. Long-term outcome after additional catheter-directed thrombolysis versus standard treatment for acute iliofemoral deep vein thrombosis (the CaVenT study): A randomised controlled trial. Lancet 379, 31-38 (2012).

16. Grunwald, M. R. \& Hofmann, L. V. Comparison of Urokinase, Alteplase, and Reteplase for Catheter-directed Thrombolysis of Deep Venous Thrombosis. J. Vasc. Interv. Radiol. 15, 347-352 (2004).

17. Shortell, C. K. et al. Safety and efficacy of limited-dose tissue plasminogen activator in acute vascular occlusion. J. Vasc. Surg. 34, 854-859 (2001).

18. Mewissen, M. W. et al. Catheter-directed thrombolysis for lower extremity deep venous thrombosis: report of a national multicenter registry. Radiology 211, 39-49 (1999).

19. Gagne, P., Khoury, T., Zadeh, B. J. \& Rajasinghe, H. A. A Multicenter, Retrospective Study of the Effectiveness of the Trellis- 8 System in the Treatment of Proximal Lower-Extremity Deep Vein Thrombosis. Ann. Vasc. Surg. 29, 1633-1641 (2015).

20. Hirsh, J. \& Hoak, J. Management of Deep Vein Thrombosis and Pulmonary Embolism . Circulation 93, 2212-2245 (1996).

21. Chiasakul, T. \& Cuker, A. The case for catheter-directed thrombolysis in selected patients with acute proximal deep vein thrombosis. Blood Adv. 2, 1799-1802 (2018).

22. Brandt, K., McGinn, K. \& Quedado, J. Low-Dose Systemic Alteplase (tPA) for the Treatment of Pulmonary Embolism. Ann. Pharmacother. 49, 818-824 (2015). 
23. Cornman-Homonoff, J., Kishore, S., Camacho, J. C. \& Winokur, R. S. Intravascular Ultrasound-Guided Extraction of Free-Floating Inferior Vena Cava Tumor Thrombus Using the ClotTriever Mechanical Thrombectomy Device. J. Vasc. Interv. Radiol. 30, 16791682.e1 (2019).

24. Benarroch-Gampel, J. et al. Technical success and short-term outcomes after treatment of lower extremity deep vein thrombosis with the ClotTriever system: A preliminary experience. J. Vasc. Surg. Venous Lymphat. Disord. 8, 174-181 (2020).

25. Bader, K. B. et al. Efficacy of histotripsy combined with rt-PA in vitro. Phys. Med. Biol. 61, 5253-5274 (2016).

26. Ngo, T. H. \& Declerck, P. J. Immunological quantitation of rabbit plasminogen activator inhibitor-1 in biological samples: evidence that rabbit platelets do not contain PAI-1. Thromb. Haemost. 82, 1510-1515 (1999).

27. Flight, S. M., Masci, P. P., Lavin, M. F. \& Gaffney, P. J. Resistance of porcine blood clots to lysis relates to poor activation of porcine plasminogen by tissue plasminogen activator. Blood Coagul. Fibrinolysis 17, 417-420 (2006).

28. Siller-Matula, J. M., Plasenzotti, R., Spiel, A., Quehenberger, P. \& Jilma, B. Interspecies differences in coagulation profile. Thromb. Haemost. 100, 397-404 (2017).

29. Huang, S., Shekhar, H. \& Holland, C. K. Comparative lytic efficacy of rt-PA and ultrasound in porcine versus human clots. PLoS One 12, e0177786--20 (2017).

30. Vedantham, S. et al. Pharmacomechanical Catheter-Directed Thrombolysis for Deep-Vein Thrombosis. N. Engl. J. Med. 377, 2240-2252 (2017). 
31. Weinberg, A. S., Dohad, S., Ramzy, D., Madyoon, H. \& Tapson, V. F. Clot Extraction with the FlowTriever Device in Acute Massive Pulmonary Embolism. J. Intensive Care Med. 31, 676-679 (2016).

32. Quinn, E., Arndt, M., Capanegro, J. \& Sherard, D. Successful removal of an acute deep vein thrombosis by the INARI ClotTriever system. Radiol. Case Reports 16, 1433-1437 (2021).

33. Yuriditsky, E. et al. Histologic assessment of lower extremity deep vein thrombus from patients undergoing percutaneous mechanical thrombectomy. J. Vasc. Surg. Venous Lymphat. Disord. 1-8 (2021) doi:10.1016/j.jvsv.2021.03.010.

34. Czaplicki, C. et al. Can thrombus age guide thrombolytic therapy? Cardiovasc. Diagn. Ther. 7, S186--S196 (2017).

35. Nosaka, M., Ishida, Y., Kimura, A. \& Kondo, T. Time-dependent appearance of intrathrombus neutrophils and macrophages in a stasis-induced deep vein thrombosis model and its application to thrombus age determination. Int. J. Legal Med. 123, 235-240 (2009).

36. Hagenah, K. CHARACTERIZATION OFTHROMBI THROUGH MRI AND HISTOLOGICAL ANALYSISOF ERYTHROCYTE GHOST CLOTS. (Pennsylvania State University, 2017).

37. Mansueto, G. et al. The dating of thrombus organization in cases of pulmonary embolism: An autopsy study. BMC Cardiovasc. Disord. 19, 1-8 (2019).

38. Mfoumou, E., Tripette, J., Blostein, M. \& Cloutier, G. Time-dependent hardening of blood clots quantitatively measured in vivo with shear-wave ultrasound imaging in a rabbit model of venous thrombosis. Thromb. Res. 133, 265-271 (2014). 
39. Chernysh, I. N. et al. The distinctive structure and composition of arterial and venous thrombi and pulmonary emboli. Sci. Rep. 10, 1-12 (2020).

40. Tan, K. T. \& Lip, G. Y. H. Red vs White Thrombi: Treating the Right Clot Is Crucial. Arch. Intern. Med. 163, 2534-2535 (2003).

41. Cines, D. B. et al. Clot contraction: Compression of erythrocytes into tightly packed polyhedra and redistribution of platelets and fibrin. Blood 123, 1596-1603 (2014).

42. Mercado-Shekhar, K. P. et al. Effect of Clot Stiffness on Recombinant Tissue Plasminogen Activator Lytic Susceptibility in Vitro. Ultrasound Med. Biol. (2018).

43. Hart, R. G., Benavente, O., McBride, R. \& Pearce, L. A. Antithrombotic therapy to prevent stroke in patients with atrial fibrillation: A meta-analysis. Ann. Intern. Med. 131, 492-501 (1999).

44. Furukoji, E. et al. CD163 macrophage and erythrocyte contents in aspirated deep vein thrombus are associated with the time after onset: A pilot study. Thromb. J. 14, 1-8 (2016).

45. Tutwiler, V. et al. Shape changes of erythrocytes during blood clot contraction and the structure of polyhedrocytes. Sci. Rep. 8, 1-14 (2018).

46. Mirshahi, M., Azzarone, B., Soria, J., Mirshahi, F. \& Soria, C. The role of fibroblasts in organization and degradation of a fibrin clot. J. Lab. Clin. Med. 117, 274-281 (1991).

47. Tracy, L. E., Minasian, R. A. \& Caterson, E. J. Extracellular Matrix and Dermal Fibroblast Function in the Healing Wound. Adv. Wound Care 5, 119-136 (2016). 
48. Mueller, H. S., Roberts, R., Teichman, S. L. \& Sobel, B. E. Thrombolytic therapy in acute myocardial infarction: Part II--rt-PA. Med. Clin. North Am. 73, 387-407 (1989).

49. Tsikouris, J. P. \& Tsikouris, A. P. A review of available fibrin-specific thrombolytic agents used in acute myocardial infarction. Pharmacotherapy 21, 207-217 (2001).

50. Hervio, L. S. et al. Negative selectivity and the evolution of protease cascades: The specificity of plasmin for peptide and protein substrates. Chem. Biol. 7, 443-452 (2000).

51. Puy, C. et al. Endothelial PAI-1 (Plasminogen Activator Inhibitor-1) Blocks the Intrinsic Pathway of Coagulation, Inducing the Clearance and Degradation of FXIa (Activated Factor XI). Arterioscler. Thromb. Vasc. Biol. 39, 1390-1401 (2019).

52. Sawdey, M. S. \& Loskutoff, D. J. Regulation of murine type 1 plasminogen activator inhibitor gene expression in vivo. Tissue specificity and induction by lipopolysaccharide, tumor necrosis factor-alpha, and transforming growth factor-beta. J. Clin. Invest. 88, 13461353 (1991).

53. Levin, E. G. \& Santell, L. Association of a plasminogen activator inhibitor (PAI-1) with the growth substratum and membrane of human endothelial cells. J. Cell Biol. 105, 2543-2549 (1987).

54. Cesari, M., Pahor, M. \& Incalzi, R. A. REVIEW: Plasminogen Activator Inhibitor-1 (PAI1): A Key Factor Linking Fibrinolysis and Age-Related Subclinical and Clinical Conditions. Cardiovasc. Ther. 28, e72-e91 (2010).

55. Baluta, M. M. \& Vintila, M. M. PAI-1 Inhibition - Another Therapeutic Option for Cardiovascular Protection. Maedica (Buchar). 10, 147-152 (2015). 
56. Liebeskind, D. S. et al. CT and MRI Early Vessel Signs Reflect Clot Composition in Acute Stroke. Stroke 42, 1237-1243 (2011).

57. Jain, V., Abdulmalik, O., Propert, K. J. \& Wehrli, F. W. Investigating the magnetic susceptibility properties of fresh human blood for noninvasive oxygen saturation quantification. Magn. Reson. Med. 68, 863-867 (2012).

58. Chang, S. et al. Quantitative Susceptibility Mapping of Intracerebral Hemorrhages at Various Stages. J. Magn. Reson. Imaging 44, 420-425 (2015).

59. Xie, H. et al. Staging deep venous thrombosis using ultrasound elasticity imaging: animal model. Ultrasound Med. Biol. 30, 1385-1396 (2004).

60. Emelianov, S. Y. et al. Triplex ultrasound: elasticity imaging to age deep venous thrombosis. Ultrasound Med. Biol. 28, 757-767 (2002).

61. Bajd, F. \& SERŠA, I. A Concept of Thrombolysis as a Corrosion-Erosion Process Verified by Optical Microscopy. Microcirculation 19, 632-641 (2012).

62. Weisel, J. W. Structure of fibrin: impact on clot stability. J. Thromb. Haemost. 5 Suppl 1, 116-124 (2007).

63. Shin, J. W., Jeong, H. S., Kwon, H.-J., Song, K. S. \& Kim, J. High red blood cell composition in clots is associated with successful recanalization during intra-arterial thrombectomy. PLoS One 13, e0197492 (2018).

64. Shaw, G. J., Sperling, M. \& Meunier, J. M. Long-term stability of recombinant tissue plasminogen activator at -80 C. BMC Res. Notes 2, 117 (2009). 
65. Hilleman, D. E. \& Razavi, M. K. Clinical and Economic Evaluation of the Trellis-8 Infusion Catheter for Deep Vein Thrombosis. J. Vasc. Interv. Radiol. 19, 377-383 (2008).

66. Kressler, B. et al. Nonlinear Regularization for Per Voxel Estimation of Magnetic Susceptibility Distributions From MRI Field Maps. IEEE Trans. Med. Imaging 29, 273281 (2010).

67. de Rochefort, L. et al. Quantitative susceptibility map reconstruction from MR phase data using bayesian regularization: Validation and application to brain imaging. Magn. Reson. Med. 61, NA--NA (2009).

68. Deng, Y., Rouze, N. C., Palmeri, M. L. \& Nightingale, K. R. Ultrasonic Shear Wave Elasticity Imaging (SWEI) Sequencing and Data Processing Using a Verasonics Research Scanner. Ultrason. Ferroelectr. Freq. Control. IEEE Trans. 1 (2017).

\section{Acknowledgements}

The authors would like to thank the University of Chicago Human Tissue Resource Center for assistance in processing the histological samples, which is supported by the Cancer Center Supplementary Grant, as well as Mr. Jude Brewer for his assistance in processing the MRI data. Funding for this project was supported in part by NIH (Grant Number R01 HL133334) and the Department of Radiology.

\section{Author Contributions}

S.A.H., A.D., A.B., E. S., D. M., G. D. W., Y. Z, S. S., and Z. F. L. performed experiments and contributed to analysis of the data. K.B.B., J.D. P., O. A., and R.O.A advised on the experimental 
design. S.A.H. wrote the first draft of the manuscript, which was critically revised by all authors. All authors approved the final version of the manuscript.

\section{Additional Information}

K.B.B. obtains funding from the National Institutes of Health (Grant Number R01 HL133334). O. A. has acted as a consultant for Inari Medical, Boston Scientific, and received research grants from Inari Medical, Canon Medical, and Philips. He acted as a speaker and received compensation for Argon Medical, Canon Medical, Penumbra, Philips, and Johnson and Johnson. G.D.W. received honoraria and serves on the advisory board for Diagnostica Stago. S.A.H., A.B., A.D., E.S., D.M., R.O.A., Y.Z., S.S., Z.F.L., J.D.P. declare no potential conflict of interest.

\section{Figure legends}

Figure 7: The fractional area of collagen, fibrin, platelets, and erythrocytes (RBC) present in (top) all analyzed VTE specimen, (bottom left) pulmonary emboli, and (bottom right) deep vein thrombi. Red crosses indicate outliers and horizontal red lines indicate median values. The top and bottom portions of the blue box represent the $25^{\text {th }}$ and $75^{\text {th }}$ percentiles, respectively, and whiskers extent to the data points not considered outliers.

Figure 8: Observed patient-to-patient variability in thrombus collagen composition $(0.06 \%$ by area for left vs. $60 \%$ for right), which appears blue in the Masson's trichrome stain. The scale bars indicate $2 \mathrm{~mm}$. 
Figure 9: Trends in thrombus composition for fibrin, collagen, red blood cells (RBCs), and platelets. Each datapoint corresponds to one thrombus sample.

Figure 10: Thrombus composition for acute ( $<7$ days old) and chronic ( $\geq 7$ days old) thrombi.

Figure 11: Representative immunofluorescent data collected from VTE. (A) Combined overlay and thrombus components analyzed using immunofluorescence, (B) erythrocytes, (C) platelets, (D) collagen, (E) fibrin, (F) PAI-1 and (G) VEGR-1. Images B-G are spectral deconvolutions of the single image A. The scale bar in image A applies to all images. Blue arrows indicate the colocation of fibrin, collagen, and platelets, respectively. The red arrow indicates a fibrin-rich border that contains almost no red blood cells. Antibody information can be found in Supplemental Table 1.

Figure 12: Example of nucleated cells present in the sample (blue) along with PAI-1 (red). Orange arrows indicate nucleated cells that are co-located with PAI-1, whereas magenta arrows indicate nucleated cells within the clot not associated with PAI-1.

Figure 7: Comparison of clot mass loss vs. quantitative susceptibility map data $(\boldsymbol{\rho}=0.90, \mathrm{p}<0.01, \mathrm{~N}=9$ samples $)$. Error bars represent the range of positive QSM values observed within the sample. One sample was highly heterogenous (red cross), and gross observation indicated strong variation between the portion of that specimen that was subjected to rt-PA and 
the portion subjected to QSM analysis. The dashed yellow line is a least-squares best fit to the data $\left(\mathrm{R}^{2}=0.77\right)$. 


\section{Supplementary Files}

This is a list of supplementary files associated with this preprint. Click to download.

- Supplementalfigures2021July21.pdf 